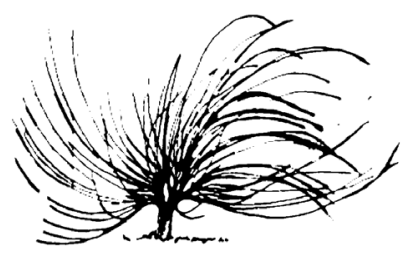

\title{
Indisciplinando la didáctica. Reflexiones sobre una experiencia filosófico- pedagógica compartida
}

\author{
Alejandro Cerletti ${ }^{1}$ \\ Universidad de Buenos Aires y \\ Universidad Nacional de General Sarmiento \\ Argentina \\ acerlett@filo.uba.ar \\ Gabriela D'Odorico ${ }^{2}$ \\ Universidad de Buenos Aires y \\ Universidad Nacional de las Artes \\ Argentina \\ gdodorico@sociales.uba.ar
}

En este trabajo se aborda la cuestión de la "Didáctica de la filosofía", elucidando, en especial, la posibilidad de una "Didáctica filosófica de la filosofía" centrada, fundamentalmente en la creación conceptual más que en la repetición o el traspaso de algunos conocimientos filosóficos canónicos, de profesores a sus alumnos. Se privilegia por tanto, la originalidad del pensar más que la reiteración de algunas recetas para aprender. Como despliegue de las ideas propuestas, se exponen algunas experiencias de enseñanza y aprendizaje filosófico, que pretenden ser coherentes con dichas ideas.

Entre los días 6 y 10 de noviembre tuvo lugar en la Universidad Nacional de Costa Rica (División Educología - CIDE) el encuentro "Enseñanza de la filosofía: indisciplinando la didáctica", al que fuimos especialmente invitados por la Universidad. El evento fue organizado por los estudiantes del Módulo Pedagógico III "Investigación e innovación educativa en la práctica docente de la enseñanza de la filosofía"

1 Doctor en Filosofía por las Universidades París 8 y Buenos Aires.

2 Doctora en Ciencias Sociales por la Universidad de Buenos Aires. 
$(2017)^{3}$, cuyo responsable es el profesor Juan Gómez Torres. Fueron cinco jornadas cuidadosamente gestionadas por el grupo de estudiantes, de una intensa actividad de ocho horas diarias en las que se tematizó la cuestión "enseñar/aprender filosofía". Desde diferentes perspectivas, se presentaron y analizaron diversas experiencias y propuestas filosófico-pedagógicas y se reflexionó sobre el sentido y las posibilidades de una didáctica filosófica. Participaron no solo los estudiantes organizadores sino también varios graduados de la universidad, profesores de escuelas secundarias y otros interesados en la temática. El encuentro fue de una enorme riqueza por la intensidad y calidad de las discusiones que se suscitaron día a día, y por las posibilidades de investigaciones futuras que quedaron abiertas, como profundización de muchas de las problemáticas abordadas. Las actividades se desarrollaron en un clima de gran cordialidad y respeto, y, sobre todo, de escucha atenta, lo que permitió transformar el encuentro académico en un verdadero taller de pensamiento filosófico compartido, muy fértil y estimulante.

Querríamos destacar, de manera sucinta, dos momentos en particular, en los que tuvimos oportunidad de ofrecer algunas ideas al debate, que nos parecieron significativas por las alternativas que abren a la reflexión.

En su participación inicial, Alejandro Cerletti eligió como eje de su exposición analizar el significado y las posibilidades de una "Didáctica de la filosofía" y, en especial, elucidar qué sería una "Didáctica filosófica de la filosofía", centrada fundamentalmente en la creación conceptual más que en la repetición o el traspaso de algunos conocimientos filosóficos canónicos, de profesores a alumnos; o sea, apoyada en la originalidad del pensar más que en la reiteración de algunas recetas para aprender.

Se sostuvo que enseñar filosofía en instituciones educativas implica ubicarse de antemano en una continuidad, que es el espacio institucional donde se enseña, el cual supone múltiples condicionamientos, la mayoría de los cuales no se es siquiera consciente, si no se lleva adelante un exhaustivo trabajo de revisión. Entonces, ¿cómo sería posible dar un lugar a lo nuevo que puede haber frente a la reproducción de lo

3 El equipo organizador estuvo integrado por Olman Alfaro Badilla, César Blanco Hernández, Sofía Cruz Icabalceta, Carlos Hernández Navarro, Kenneth Hernández Víquez, Adrián Mata Calderón, Daniel Porras Navarro, Oscar Rivas Monge, Luis Rodríguez Soto, María José Romero Castro, Sergio Ruíz Zamora, Omar J. Ureña Soto y Jean Carlo Víquez Navarro. 
existente, que, parece ser, la inexorable secuencia de la transmisión institucional? ¿Desde dónde puede ser esto pensado filosóficamente? ¿Es posible una "didáctica" de la filosofía o una didáctica filosófica, que sea coherente con lo anterior?

El hecho de que la filosofía se ocupe filosóficamente de su enseñanza ha significado tener que construir la cuestión "enseñar filosofía", en primer lugar, como un problema filosófico. Responder filosóficamente a la pregunta "¿qué es enseñar filosofía?" supone una caracterización previa de la filosofía, se la haga evidente o no, ya que esta va a estar implícita tanto en el modo de responder (en la medida en que se va a responder "filosóficamente") como en el contenido u objeto de la pregunta (en tanto se inquiere sobre enseñar, justamente, "filosofía"). Cuando no se aclara esta caracterización de la filosofía adoptada, se enseña y aprende a enseñar filosofía desde la naturalización de alguna idea de filosofía y de alguna concepción de enseñanza y aprendizaje. En ese caso, habría una idea de filosofía supuesta, que no se cuestiona, al menos explícitamente, y que opera como la referencia filosófica del enseñar y aprender. Esto es lo que suele conformar la normalidad de la transmisión educativa.

Por este motivo, para abordar desde la filosofía su propia enseñanza, correspondería explicitar, en primer lugar, la orientación filosófica desde la cual se va a interrogar esa enseñanza. En nuestro caso, nos resultó útil abordar estas cuestiones desde filosofías que tengan su preocupación teórica central en la novedad y el acontecer; que privilegien la dimensión azarosa y creativa de los encuentros (entre personas, entre pensamientos, entre saberes) y que valoren una voluntad de emancipación e igualdad, en todos los planos, pero, en especial, en la función educativa. Los filósofos que nos han ayudado en esta tarea son Alain Badiou, para fundamentar la perspectiva del acontecimiento y el sujeto; Rancière, para la igualdad y la emancipación; $\mathrm{y}$, en alguna medida, el último Althusser, con el llamado materialismo del encuentro o materialismo aleatorio.

La posibilidad de una didáctica filosófica - esto es, reiteramos, una didáctica asociada a una concepción de filosofía- lleva la intención de promover, permitir o estimular aprendizajes filosóficos. Pero, antes que nada, y para ir por partes, ¿quién aprendería filosofía, filosóficamente? ¿Cómo se constituye ese "sujeto" del aprendizaje filosófico? ¿Y qué significa ese sujeto desde una orientación filosófica como la elegida? Badiou 
ha asociado el concepto de sujeto con la alteración de una secuencia de normalidad. Es decir, desde esta perspectiva, no habría "sujeto" de una continuidad o de una reproducción. En este caso, cabría hablar más bien de "objetos" de la reproducción o la continuidad. Serían solo "objetos" porque habría "alguien" (un individuo o un conjunto de individuos) que es instalado en el ordenamiento de una maquinaria de repetición de lo existente, que intenta funcionar de manera eficiente; la institución educativa, por ejemplo. El proceso de inclusión de los individuos en esa maquinaria de repetición es, más que una subjetivación, una objetivación, porque se los conduce a formar parte del estado normal de las cosas. $\mathrm{Si}$, por el contrario, se adopta la perspectiva de que el sujeto aparece en la alteración o modificación de lo esperado - es decir, es acontecimiental, y no una objetivación disimulada-, el panorama es diferente.

Se puso énfasis en que uno de los aspectos más significativos de una alteración en lo establecido, en un contexto de enseñanza es la emergencia del pensar de los otros (del otro del docente, o de la institución). El pensamiento de los otros es algo que, por cierto, no puede ser programado. Se afirmó, de manera consecuente, que pensar significa intervenir de una manera propia en los saberes o los relatos que se ofrecen o circulan. Quien piensa sobre los conocimientos establecidos no los repite mecánicamente, sino que los recrea y apropia, a su manera. Valorar esta circunstancia implica concebir la enseñanza, y la educación en general, desde la perspectiva de lo que acontece (fundamentalmente, el pensamiento), más que desde el cumplimiento administrativo de lo planeado (la reproducción de contenidos estandarizados). Pero este enfoque supone visualizar una aparente paradoja: para que se produzca una alteración debe haber algo que pueda ser alterado. Eso suele ser el plan de enseñanza elaborado para el encuentro educativo. Lo nuevo irrumpe en lo que se había organizado, porque altera lo que había sido establecido como hipótesis de aprendizaje de los alumnos. Si esto no ocurriera, no habría novedad. Lo organizado o planeado se transforma entonces en condición de posibilidad de su propia disrupción. Toda creación necesita un encuadre, para ser desbordado.

Remarcar la importancia de la alteración de lo previsible implica adscribir a una idea de "aprender" que atribuye un lugar central a quien aprende en la construcción de su propio aprendizaje. Esto se vincula también a la noción de emancipación intelectual de Rancière, con la posibilidad de asociar o disociar lo recibido, de manera original. 
Por lo tanto, el concepto de educación subyacente en esta perspectiva está relacionado con el de autoeducación y el de enseñanza con el de autoaprendizaje. Se adoptó, consecuentemente, una caracterización genérica de educar, y por extensión de enseñar, como aquella actividad que permite disponer ciertos saberes y prácticas para que puedan ser interpelados y apropiados; es decir, para que puedan ser, en sentido estricto, pensados y actuados.

Toda situación de enseñanza implica encuentros; encuentros múltiples de múltiples factores, que son imposibles de prever en toda su extensión. Y todo encuentro tiene una dimensión aleatoria; siempre está acechado por lo impredecible porque nunca se puede saber con certeza qué es lo que va a suceder o qué efectos se pueden producir, si la voz del otro importa. El "sujeto" de esos encuentros no es, en sentido estricto, un sujeto individual (tal o cual alumno, ese profesor, etc.), porque en el movimiento del enseñar-aprender está involucrado un conjunto complejo de multiplicidades: estudiantes, maestros o profesores, saberes, lugares, tiempos, sueños, miedos, amores. Se trata más bien de un sujeto que involucra a todos, es decir, es colectivo, porque hay un vínculo compartido, o un común, que se actualiza clase a clase. En el caso específico de la filosofía, ese sujeto colectivo es tradicionalmente un sujeto dialógico. El quiebre de la unidireccionalidad del discurso escolarizado hace que puedan emerger otros discursos, otras palabras. La convergencia dialógica de las novedades que portan quienes participan de esos encuentros establece un lugar de subjetivación que es común a esos participantes. Podríamos llamarlo también un colectivo dialógico o una comunidad de diálogo. Este sería el "sujeto" preponderante de una clase filosófica, ya que nace en la construcción común de un diálogo (entre personas, saberes, pensamientos).

Que el sujeto de una situación de enseñanza y aprendizaje filosófico sea, básicamente, un sujeto colectivo significa que lo que se constituye en sujeto es verdaderamente la relación (docentes-alumnos-saberes) en su conjunto, ya que se produce una recomposición del vínculo a partir de la novedad de cada uno. Solo por extensión, quienes participan de ese sujeto (colectivo) podrían ser a su vez llamados sujetos (individuales). Asimismo, el sujeto es colectivo porque hubiera sido otra la recomposición (o no hubiera ocurrido nada) si hubiesen sido otros los participantes y otro el contexto. Es decir, la posibilidad de que exista un sujeto depende de una particular combinación de circunstancias y 
decisiones en una situación y no de una práctica pedagógica instituida, una programación o una política educativa. Quienes forman el sujeto filosófico colectivo son los reales protagonistas del acto de enseñar y aprender filosofía. Se es partícipe de la propia formación filosófica a partir de una decisión sobre el encuentro con los saberes y con los otros.

En consecuencia, en el marco de una didáctica filosófica -en el sentido que se ha tratado de darle-, para que podamos calificar a una clase de filosófica deberá ocurrir algo diferente de la mera presentación (exposición, explicación, etc.) por parte de un profesor de un tema de la filosofía. Una clase filosófica será aquella en la que se pone algo en juego (un texto, un problema) que debe ser reapropiado por quienes intervienen en la clase. Esta reapropiación, al posibilitar la intervención creativa de los participantes, tiene una dimensión azarosa, ya que lo que se despliega es el pensar. Y, como fue señalado, el pensar excede siempre la repetición o el traspaso de un conocimiento. Esta irrupción aleatoria excede cualquier planificación, pero se necesita de una planificación que valore esa circunstancia, para darle lugar en caso de que ocurra. El pensamiento no es una digresión o una falla del plan inicial sino la consumación de la filosofía. Si se considera que cualquier alteración en el plan inicial debe ser anulada para forzar el cumplimiento administrativo de lo que el profesor (y el programa) tenía que decir, se privilegia la traslación unilateral de un conocimiento, sin intervención creativa de quienes lo "reciben".

La acción docente debería ser disponer los saberes filosóficos existentes de una manera especial, de modo tal que puedan ser interpelados subjetivamente, para que quizás tenga lugar un pensar. El maestro filósofo o el profesor tienen la tarea de introducir desafíos intelectuales y prácticos en el contexto de un grupo de aprendizaje. Se trata de una decisión filosófica, política y didáctica. Hay siempre algo de incomodidad en la intervención del pensamiento porque implica intentar un acceso diferente a lo ya sabido. En la enseñanza repetitiva el "lugar" del estudiante es el no-lugar del encuentro. Su participación solo es solicitada eventualmente para confirmar que la repetición está siendo eficaz.

En el contexto de una didáctica del encuentro y del acontecer la tarea del profesor es múltiple, ya que debe al mismo tiempo provocar, acompañar, transmitir, estimular y, sobre todo, saber escuchar y saber aprender de y en sus clases. Debe situar una continuidad de transmisión de saberes y prácticas filosóficas sobre las que se provocará el 
pensamiento a partir de interrogantes y problematizaciones. Y en el mismo movimiento, tiene que estimular el pensar, alentar la construcción compartida de interrogantes y la búsqueda de respuestas, individuales y colectivas; es decir, favorecer el recorrido de trayectos singulares.

Las situaciones de enseñanza filosófica permitirán encuentros en los cuales podrá ocurrir algo (o tal vez nada). De esos encuentros participarán docentes, estudiantes y saberes, en un contexto específico. Cada situación organizada de enseñanza supone una repetición y la posibilidad de su alteración. Que haya sujetos estará vinculado íntimamente con la irrupción de algo que interpele los saberes previos de una manera singular y transforme a quienes han participado de ese encuentro.

La didáctica de la filosofía es un conjunto de supuestos y de decisiones formales respecto de la tarea de enseñar filosofía. Cada situación particular de enseñanza filosófica requiere una actualización de esas decisiones con el objeto de construir un campo de propuestas. Esto significa que las propuestas que se lleven adelante en una clase y lo que con ellas ocurra constituirán una singularidad, efecto de una combinación de elementos irrepetibles: el grupo de alumnos, el profesor, las condiciones de la clase, el contexto.

Una didáctica de la filosofía que privilegia la contingencia de los encuentros y que adquiere su sentido en la potencialidad del aprendizaje merece llamarse "aleatoria", en la medida en que no está limitada por un resultado específico preconcebido. Los resultados son una construcción compartida del proceso de aprendizaje, que depende, en gran medida, de la singularidad de la situación educativa.

La didáctica aleatoria es también, en un sentido preciso, una meta-didáctica, porque constituye un horizonte de posibilidad de didácticas específicas de la filosofía. Podría concebirse como una suerte de "principio filosófico-didáctico" que, como tal, no prescribe acciones particulares sino más bien ofrece una referencia para recorrer diversos caminos. Es el mínimum de una filosofía enseñable, que privilegia el filosofar de los alumnos. Por cierto, se trata de una meta-didáctica porque parte de una hipótesis general (y condicional): es posible aprender y enseñar filosofía si se asume que toda enseñanza de la filosofía es definida por el aprendizaje filosófico, y que todo encuentro tiene una dimensión repetitiva (la filosofía y los conocimientos previos puestos en juego) y otra adversa, que será el foco de una creación por parte de quienes aprenden y enseñan; en esto radica el filosofar compartido. 
La didáctica aleatoria no concierne en un nivel educativo especial porque es básicamente una hipótesis de aprendizaje filosófico unido a una voluntad de enseñar. Cada nivel tiene sus particulares requerimientos institucionales, por lo que en cada situación se deberán construir los procedimientos específicos para llevarla adelante.

Una didáctica filosófica aleatoria se va a situar en un lugar institucional incómodo, porque interpela la continuidad de la transmisión administrativa de los saberes. El hecho de que las situaciones de enseñanza tengan lugar sobre lo fortuito de los encuentros quiere decir que cada clase expresa una compleja confluencia de personas, saberes, normas, tradiciones institucionales, deseos, en la que se establecen múltiples relaciones que deben actualizarse a diario. En cada una de las clases tiene lugar un encuentro siempre expuesto a lo inesperado, siempre abierto a lo que pueda ocurrir ${ }^{4}$.

A continuación, en una segunda instancia del encuentro, tomamos como punto de partida para la reflexión colectiva algunas prácticas específicas de enseñanza de la filosofía presentadas por Gabriela D'Odorico. Nuestras referencias funcionaron como un escenario para pensar la relación entre la enseñanza de la filosofía y los vínculos puestos en juegos en los espacios para la reflexión. Quisimos someter a la discusión diferentes posibilidades de construcción de lo común a partir de prácticas de la enseñanza del pensamiento filosófico en espacios institucionales como la escuela media y la universidad.

Una de las experiencias evocadas fue la de un seminario de Antropología Filosófica dictado durante varios años en profesorados de Filosofía de la Ciudad de Buenos Aires en la República Argentina. Los cursantes de dicho seminario son futuros profesores de esta disciplina que ya realizaron o estaban realizando sus prácticas docentes en la escuela secundaria. A su vez, integran el seminario estudiantes que ya lo han cursado y que se incorporan en carácter de ayudantes-alumnos. También participan graduados recientes que, en condición de investigadores adscritos, se especializan en la enseñanza de alguna problemática concerniente a la antropología filosófica.

4 En la descripción de este primer momento, han sido utilizados algunos fragmentos del artículo de Alejandro Cerletti "Didáctica aleatoria de la filosofía, dialéctica del aprendizaje filosófico", publicado en Cerletti, A. y Couló, A. (orgs.). (2015). Didácticas de la filosofía. Entre enseñar y aprender a filosofar. Buenos Aires: Noveduc, pp. 15-32, modificados parcialmente para este trabajo. 
El seminario propone la dinámica de un taller de reflexión y de escritura filosófica orientado a elucidar los supuestos antropológicos que operan en las propias prácticas docentes de quienes dictamos materias para esta especialidad. Buscamos así sacar a la luz los usos de concepciones habituales acerca de lo normal, lo humano, del cuerpo, de la vida, de la muerte, de la raza, de la etnia o del género con las que nos desempeñamos como profesores de filosofía. Asumimos que la actividad en la que nos embarcamos está atravesada por la incertidumbre y por la fragilidad inherente a un proceso que atiende, especialmente, a la construcción colectiva. Sin embargo, nuestra apuesta está anclada en la convicción de que una voluntad de sospecha se forja reflexionando sobre las modalidades que asumen los procesos de producción, de circulación y de uso de los conocimientos frente a los problemas sociales y políticos propios de nuestro presente.

Los practicantes y pedagogos recientes que arriban a las escuelas secundarias de Buenos Aires y sus alrededores, año tras año, manifiestan algunas preocupaciones, muchas veces, reiteradas en las diferentes instituciones. Entre ellas genera gran inquietud un presupuesto bastante extendido: las funciones y responsabilidades asignadas a los profesores de filosofía en el ámbito de la escuela media. Durante el transcurso del seminario caracterizamos cierto sentido común institucional acerca de lo que significa enseñar filosofía porque lo encontramos repetido en gran parte de las escuelas en las que instruimos. Los colegas que dictan otras asignaturas, directivos, administrativos y buena parte de los estudiantes entienden que los profesores de filosofía se ocupan $-\mathrm{y}$ si no lo hacen deberían hacerlo- casi específicamente de la formación "humana" de los jóvenes. Esta función de la filosofía en la vida de los adolescentes vendría no solo a compensar sino también a dar batalla al avasallante proceso de tecnologización de la vida que trae a nuestras aulas contradicciones, muchas veces difíciles de manejar en las clases. Para este sentido común institucional, la virtualización de los materiales de estudio o el uso didáctico de dispositivos de comunicación, a la vez que facilita procedimientos, implica la despersonalización y la deshumanización de los vínculos. El problema se acentúa cuando los docentes comenzamos a advertir el impacto, incluso en el campo de la educación, de la tecnología biológica, médica y farmacológica que busca instaurar parámetros de "normalidad" física, psíquica y social. 
En este contexto, se construye el sentido común de las instituciones de nivel medio que interpretan como tarea fundamental de los profesores de filosofía la formación humana -humanización- de estudiantes que deben lidiar a diario con la multiplicación indefinida de contenidos y de novedades específicas que animan al resto de las asignaturas. La creciente profesionalización y especialización del conocimiento, presente en los planes de estudio, parece delegar la responsabilidad de la formación humana, principalmente, a los profesores de filosofía.

A partir de esta caracterización los miembros del seminario nos propusimos interrogar la ambigüedad que advertimos en esta demanda de "humanizar" a los jóvenes. Nos interesó indagar cuán potente para el pensamiento es esa función que, según parece, tenemos encomendada los profesores de filosofía. Debíamos discutir si ese reclamo obedece a la necesidad de "refuncionalizar" la enseñanza de la filosofía, es decir, de hacerla compatible y tal vez someterla a las necesidades del actual desarrollo tecnocientífico. O bien se trata de un intento de colocar esta enseñanza a la cabeza de un proyecto de humanización que rescata viejos y debilitados valores culturales o religiosos. Esas primeras hipótesis que examinamos no contemplaban la capacidad de autogestión y la autonomía de los profesores y estudiantes que hacen posible las clases de filosofía. Sin embargo, en nuestros trabajos colectivos, extendimos la interrogación a los supuestos acerca de lo humano con que nosotros recibimos esa demanda, tratamos de evaluar el reclamo como un síntoma de un problema más complejo que debíamos reconstruir.

Entonces teníamos dos cuestiones interrelacionadas para trabajar, una era el diagnóstico del mundo contemporáneo en el que habitamos como un paisaje tecnológico que nos modifica a la vez que nosotros lo modificamos. La otra era la concepción acerca de lo humano que surge en compatibilidad y adaptación a ese entorno.

Para bosquejar un escenario posible en el cual situar la enseñanza de la filosofía recurrimos a producciones de la literatura, muchas de ellas llevadas al cine, a las series televisivas, al teatro y a la danza. Se trata de obras en las que el entorno, sea natural o tecnológico, es analizado a partir de su fusión con la percepción humana. La naturaleza y los paisajes artificiales que proliferan en grandes urbes también nos transfiguran, conforman gustos y pasiones, diseñan químicamente nuestros estados de ánimo y son tema de buena parte de la literatura en el último 
siglo. Entendemos que la literatura es un modo de pensamiento que se propuso indagar en profundidad sobre esta problemática y que debíamos abordarla sin la pretensión de ilustrar conceptos, ni utilizarla como un mero instrumento de traducción de los desarrollos filosóficos.

Con el inicio de cada ciclo lectivo compilamos una antología de textos literarios de un escritor que nos va a acompañar durante el curso, un autor que entrará en diálogo con el material filosófico del programa del seminario y nuestras prácticas docentes. En los diferentes ciclos tuvimos recensiones de las discusiones, material pedagógico, textos de escritura colectiva, ensayos filosóficos, videos e, incluso cuentos, afines a la temática de la antropología filosófica. Nuestra atención se concentró en detectar las singularidades que cobra la concepción de lo humano en los diferentes entornos -naturales o tecnológicos- en los que la literatura de ese ciclo nos sitúa. Tuvimos años dedicados a Franz Kafka, Jean-Paul Sartre, James Graham Ballard, Italo Calvino y Michel Tournier. También hubo ciclos consagrados a escritores argentinos como Lucio V. Mansilla, Jorge Luis Borges, Carlos Correas o Juan José Saer. En estos autores es posible detectar personajes de maestros, profesores de escuela media, académicos universitarios, situaciones de enseñanza-aprendizaje y variadas referencias filosóficas.

Nuestro análisis literario interroga especialmente las posibilidades de transformación del entorno material en el que transcurren nuestras vidas, los dilemas que plantea su manejo, su explotación mercantil y la idea de progreso gestada en la modernidad. La preocupación deriva en el reiterado blanco de análisis de los autores que son las fronteras interiores, los límites entre la imaginación y la percepción del entorno. Los acontecimientos se dan en universos virtuales, en tierras desconocidas, en campos de objetos que se vuelven siniestros, en entornos no reconocibles. La construcción de escenarios ficcionales, aparentemente confundidos con una naturaleza asfixiante o con una atmósfera tecnológica, nos permitió identificar encuentros y tensiones entre literatura y filosofía, ambas entendidas como dos lógicas o modos diferentes del pensar. Ambas aparecen como dos manifestaciones del pensamiento, que se ponen en juego, dialogan y se interrogan recíprocamente para la construcción de problemas.

En este marco encontramos que la enseñanza de la filosofía admite ser interpretada como una poética del saber, ateniéndonos al uso que hace Jacques Rancière de ese concepto. Esto significa que enseñar 
filosofía tiene una dimensión creativa -poética- que involucra tanto la interacción colectiva como la práctica de la escritura. Esta experiencia en la que la filosofía se confronta con la literatura, en la que la literatura aparece como un "otro" para el pensamiento filosófico, abre una serie de interrogantes acerca de su enseñanza interpretada, a su vez, como una poética o práctica creativa. Esa poética habilita, fundamentalmente, un proceso creativo de autotransformación de quienes participamos de la enseñanza-aprendizaje de la filosofía y de la gestación de un espacio que haga posible esa metamorfosis. Este trabajo de transformación no puede pensarse para individuos aislados capaces de recordar saberes aprendidos sino para formaciones colectivas que devienen autónomas respecto de los condicionamientos y sujeciones.

Nuestro trabajo reflexivo nos permitió retomar la demanda de humanización que el sentido común institucional desliza hacia la filosofía. En una primera instancia la reclamada humanización que debiéramos autoimponernos e imponer a nuestros estudiantes puede identificarse como una adaptación a criterios y convicciones de normalidad, compatibles con los procesos de tecnologización de la vida. Hay una perspectiva tecnocientífica de lo humano que vino produciendo taxonomías, clasificaciones y grillas de inteligilibilidad alrededor de ciertas categorías como género, especie, humanidad, raza o etnia, entre otras. Son ordenamientos jerárquicos que no contemplan ni las particularidades, ni las existencias singulares, ni el hecho de que el pensamiento colectivo pueda desmontarlas. La posibilidad de deshacer las concepciones de raza o género dio lugar una profusa escritura filosófica que nos invita a reflexionar sobre otras categorías clasificatorias. Allí es donde la enseñanza de la filosofía puede, entonces, interrogar, exceder y hacer estallar estas normatividades ancladas en supuestos antropológicos.

Tal vez la demanda de humanización responda con temor a un futuro distópico en el que los mecanismos de dominación y expoliación se manifiesten de manera tecnocientífica y, por ello, rememore perspectivas antropológicas moralizantes y religiosas del pasado. Pero también podemos interpretar que esa demanda nos obliga a pensarnos filosóficamente dentro del actual proceso de tecnologización del entorno y de la vida que arroja nuestra existencia en un mundo que nos aparece cada vez menos comprensible. Sumergidos en esa incomprensión que podría atentar contra la dimensión creativa de nuestra tarea docente podemos 
construir nuestra libertad y autonomía en la horizontalidad de la reflexión compartida.

Otra de las experiencias evocadas en el encuentro "Indisciplinando la didáctica" tuvo como eje la enseñanza de la filosofía en carreras universitarias artísticas, en particular, en las orientaciones de danza, de teatro y de música. La implementación de la asignatura Filosofía bajo la forma de un taller con artistas que están atravesando su formación universitaria trae nuevas perspectivas problemáticas en relación con la tecnología. En las carreras artísticas, la formación académica heterogénea que aportan los cursantes hace confluir interrogantes provenientes del arte, de la filosofía y de la tecnología. La particular situación, consistente en identificar y reconstruir problemas compartidos para las diferentes prácticas profesionales, permite cavilar sobre el devenir artístico en tanto modalidad de pensamiento. A la vez, esa situación funciona como plataforma común para implicar al arte en un diálogo fluido con la filosofía y la tecnología.

En el año 2016, en Barcelona hubo una muestra artística denominada "+ Humanos. El futuro de nuestra especie". Allí, el músico y activista inglés Neil Harbisson, el primer cyborg de la historia reconocido como ciudadano, expuso su obra "Cabeza sonocromática". Harbisson padece una enfermedad congénita que le impide ver colores y se implantó en su cabeza un sensor que transforma los colores en música. Con ese dispositivo el músico compone y difunde sus obras. Pero cuando Harbisson quiso renovar su pasaporte británico el Estado se lo impidió hasta que se extrajera el implante para poder realizar la fotografía del documento. Sin embargo, a partir de su activismo cyborg logró la renovación del documento con la fotografía de su cabeza implantada.

Frente a este tipo de obra surgen muchas preguntas acerca de la manera en que el arte puede involucrar a la tecnología en sus creaciones y cómo se ven reformados los modos de composición, diseño y curaduría de las nuevas expresiones artísticas. Por otra parte, cómo determinar si ciertos artefactos son meros dispositivos tecnológicos, obras de arte, intervenciones performáticas o políticas. Lo único que se vislumbra con cierta claridad es que la complejidad de algunos problemas contemporáneos no admite un tratamiento reducido a la especificidad de alguna disciplina en particular.

Entendemos que la filosofía puede propiciar una reflexión que se sitúa en los umbrales del arte, de la política y de la tecnología. Desde esa 
situación, los problemas contemporáneos pueden ser interpretados como espacios, umbrales o zonas de indistinción entre arte, filosofía y tecnología que interrogan especialmente nuestras prácticas de investigación, de enseñanza, de evaluación y de acreditación. Con ello, interpretamos que el abordaje multidisciplinar de las nuevas problemáticas reclama la puesta en diálogo de diversas modalidades del pensamiento como son el filosófico, el artístico o el científico. Así, la necesidad de dicho coloquio se extiende a la práctica docente en filosofía, en particular de la estética y la ética, dirigida a los estudiantes de arte.

La filosofía contemporánea interactúa de un modo creciente con manifestaciones artísticas actuales en las que la tecnología no es solo un instrumento o insumo para producir obras. Debido al actual desarrollo de la biología sintética, de la ingeniería genética y de la neurobiología podemos encontrar nuevos fenómenos artísticos que se denominan bioarte, arte genético o transgénico. Se trata de artistas que utilizan los espacios y los materiales de los laboratorios biotecnológicos para producir objetos estéticos, performances e instalaciones. Los artistas trabajan con el cultivo de células, organismos genéticamente modificados, intervención sobre el ADN o tejidos vivos. De esa manera pueden crear obras vivientes que crecen, proliferan y que hay que desconectar de un biorreactor que las mantiene vivas cuando las exposiciones públicas terminan.

Podríamos decir que el bioarte aparece como una producción cultural paradigmática situada en los umbrales del arte, de la ciencia, de la tecnología y de la filosofía, en particular por los aspectos éticos y antropológicos que aparecen involucrados. ¿Qué significa que un investigador de un laboratorio biotecnológico pueda crear piezas vivientes y difundirlas como obras de arte? ¿Qué estatuto tienen la vida y la muerte en las obras con artefactos vivos asistidos biotecnológicamente que deberán ser desconectados con la finalización de la muestra? ¿Cuál es la novedad que aporta al campo de la estética un cuerpo transformado con intervenciones biomédicas e informáticas? Frente a estas preguntas que aparecen como problemas comunes para las diferentes disciplinas, podemos identificar algunas cuestiones que muestran la particularidad del abordaje filosófico. La enseñanza de la filosofía se enfrenta a problemas multidisciplinares y, a la vez, propone su modo de abordaje -filosóficopara esos problemas. Nuestras preguntas nos permiten hacer algunos señalamientos importantes. 
En primer lugar, la filosofía contemporánea interactúa de un modo creciente con manifestaciones artísticas en las que la tecnología ya no puede ser tomada como una mera herramienta o material de trabajo, tampoco es solo una concepción del mundo. Habitamos un espacio urbano-tecnificado en el que la tecnología, a su vez, nos habita cuando nos provee de dispositivos que diseñan nuestros modos de comunicación o cuando nos medicaliza para incluirnos en parámetros de normalidad. Esto equivale a mostrar que, si bien la tecnología "nos habla", "nos piensa" o "nos actúa" también, nos da herramientas para luchar por nuestra autonomía.

En segundo lugar, la experimentación científica ilimitada y el juego creativo con materiales vivientes pueden ser "modos de hacer" que se confunden, al punto de volverse indiscernibles. En ese sentido las obras de arte genético o transgénico plantean la paradoja de habilitar un "campo de experimentación científica" regido por valores estéticos -no éticos- y ser, a la vez, una práctica de visibilización, denuncia y comunicación pública de lo que ocurre en los laboratorios biotecnológicos.

Cuando el bioarte nos sitúa en el cruce entre arte, tecnología y filosofía significa que las obras, las performances y las instalaciones terminan ubicadas en una zona liminal entre lo vivo y lo muerto, entre lo viviente y las cosas inertes, entre la vida reproductiva y las mercancías. Los interrogantes en torno al estatuto de la vida, de lo orgánico, del cuerpo o de lo humano que pueden plantearse a partir del bioarte actualizan cuestionamientos de larga data para la historia de la filosofía. Reconstruir los abordajes filosóficos para problemas que atraviesan varias disciplinas es un modo de generar nuevos vínculos intersubjetivos.

Ambas experiencias evocadas en este segundo periodo del evento están atravesadas por una misma inquietud ¿qué modalidades de lo común emergen de estos espacios reflexivos y cuáles son los principales obstáculos para la indagación filosófica? Vivimos en comunidades que poseen saberes y valoraciones acerca de la filosofía y en especial, en Latinoamérica, hay experiencias comunitarias postergadas que nos obligan a volver a reflexionar sobre la vida habitual. El sentido común de nuestras comunidades, especialmente las institucionales, apela a un común que reconoce un lugar y un valor para la enseñanza de la filosofía. Y aunque no siempre es legitimada por las políticas educativas, la práctica de su instrucción ofrece abordajes diversos para dilemas no siempre novedosos. Nuevas materialidades y formatos inéditos 
conducen, muchas veces, a problemas filosóficos que fueron planteados hace miles de años. Enseñar filosofía recreando problemas para pensarlos reiteradamente en forma colectiva es también inventar un distinto modo de hacer común.

Creemos que el encuentro "Indsciplinando la didáctica" fue una exitosa práctica colectiva de deliberación filosófica con la que el hacer común salió fortalecido. 